\title{
CARACTERIZAÇÃO BROMATOLÓGICA DE FRUTOS E GELEIAS DE AMORA-PRETA ${ }^{1}$
}

\author{
ANGELA VACARO DE SOUZA², RAMON JULIANO RODRIGUES ${ }^{3}$, ESTEVÃO PERIN GOMES ${ }^{4}$, \\ GABRIEL PERIN GOMES ${ }^{5}$, ROGÉRIO LOPES VIEITES ${ }^{2}$
}

RESUMO-A amora-preta é um fruto de alta perecibilidade e por isso seu aproveitamento é preferencialmente industrial. Entretanto, há pouca informação na literatura a respeito do efeito do processamento de produtos elaborados a partir da amora. Neste trabalho, foram avaliados frutos e geleias em três estádios de colheita diferentes. O objetivo foi realizar a caracterização bromatológica, a quantificação de pectina total e solúvel e de macro e micronutrientes dos frutos e geleias. Os resultados mostraram que a amora-preta apresenta alto potencial para industrialização, pois em grande parte dos compostos avaliados, não houve grandes alterações decorrentes do processamento.

Termos para indexação: Rubus sp, geleia, processamento de alimentos.

\section{BROMATOLOGICAL CHARACTERIZATION OF BLACKBERRY FRUITS AND JELLIES}

\begin{abstract}
The blackberry fruit is highly perishable and therefore its use is preferentially industrial. However, there is few information in the literature about the effect of products processing made of blackberry. In this study, it was evaluated blackberry fruits and jellies from three different stages of harvest. The aim was the bromatological, quantification of total and soluble pectin and macro and micronutrients from fruits and jellies. The results showed that the blackberry presents a high potential for industrialization since most of the compounds evaluated there were no big changes resulted from the processing.
\end{abstract}

Index terms: Rubus sp., jelly, food processing.

\section{INTRODUÇÃO}

A amoreira-preta apresenta frutos de alta qualidade nutricional e valor econômico significativo (ANTUNES, 2002), contém teores consideráveis de vitamina $\mathrm{C}$ e água, contém cerca de $10 \%$ de carboidratos, elevado conteúdo de minerais, vitaminas do complexo B e A, além de ser fonte de compostos funcionais. A amora-preta é considerada uma fruta funcional, ou seja, além das características nutricionais básicas, quando consumida como parte usual da dieta, produz efeito fisiológico/metabólico benéfico à saúde humana, sendo segura para consumo sem supervisão médica (VIZZOTO, 2008), sendo boas fontes de antioxidantes naturais (KOCA; KARADENIZ, 2009), como as antocianinas (DEIGHTON et al., 2000) e os polifenóis (WANG; LIN, 2000; MOYER et al., 2002).

Além do consumo in natura, os frutos da amoreira-preta possuem grande potencial para serem processados como polpa, geleias, sorvetes, entre outras. A produção de geleias é uma alternativa para utilizar frutas fora do padrão de qualidade para consumo in natura, contribuindo para minimizar as perdas pós-colheita, além de agregar valor a esta matéria-prima que possui safra definida nos meses finais do ano e baixa ou nenhuma produção nos meses subsequentes.

Por essas características, a amora-preta vem despertando interesse de produtores e consumidores, principalmente pelo potencial de consumo associado às suas propriedades benéficas à saúde; no entanto,

${ }^{1}$ (Trabalho 037-14). Recebido em: 23-01-2014. Aceito para publicação em: 16-10-2014.

${ }^{2}$ Universidade Estadual Paulista, Departamento de Produção Vegetal - Horticultura - Rua José Barbosa de Barros, ${ }^{\circ} 1780$ - Jardim Paraíso - Botucatu - SP, Brasil. E-mail: angelavacaro@hotmail.com; vieites@fca.unesp.br

${ }^{3}$ Universidade Estadual Paulista, Departamento de Ciências Biológicas, Assis-SP, Brasil. E-mail: ramonjuliano@hotmail.com

${ }^{4}$ Faculdade Eduvale de Avaré - Avenida Misael Eufrásio Leal, no 347 - Avaré- SP, Brasil. E-mail: estevopg@hotmail.com

${ }^{5}$ Universidade Estadual Paulista, Departamento de Engenharia Rural -Irrigação e Drenagem -Rua José Barbosa de Barros, no 1780 -

Jardim Paraíso - Botucatu - SP, Brasil. E-mail: gabrielp.gomes@fca.unesp.br 
existem poucas pesquisas sobre seu valor nutricional e potencial industrial (HARBORNE; WILLIAMS, 2000). Diante dos dados apresentados, o presente trabalho teve como objetivo realizar a caracterização bromatológica e de minerais dos frutos e geleias.

\section{MATERIAIS E MÉTODOS}

Os frutos de amora-preta da cultivar Tupy foram colhidos manualmente em uma fazenda comercial no município de Cerqueira CésarSP (latitude $23^{\circ} 02^{\prime} 08^{\prime \prime} \mathrm{S}$, longitude $49^{\circ} 09^{\prime} 58^{\prime}$ " $\mathrm{O}$ e altitude de 737 metros), e transportados imediatamente ao Laboratório de Frutas e Hortaliças do Departamento de Horticultura, da Faculdade de Ciências Agronômicas da UNESP, Câmpus de Botucatu.

No Laboratório, os frutos foram lavados em água corrente. Após a lavagem, foram imersos por 20 minutos em solução clorada a 20 ppm e, em seguida, expostos ao ar para secarem naturalmente em bancadas de superfície lisa.

Os pontos de colheita que constituíram os tratamentos foram definidos visualmente, sendo eles: $100 \%$ vermelhas - T1; 50\% vermelhas e $50 \%$ pretas - T2 e 100\% pretas - T3. Este método de colheita foi escolhido por ser o mais utilizado pelos produtores para este tipo de fruta, sendo os frutos avaliados na ocasião da colheita.

Para a fabricação das geleias, os frutos foram primeiramente despolpados em despolpadeira de aço inoxidável descontínua, com peneira de malha de $0,5 \mathrm{~mm}$.

As geleias foram preparadas com 50:50 (fruta: açúcar cristal comercial). Para a geleificação, foi utilizada pectina comercial $(0,5 \%$ em relação ao peso da polpa), não sendo adicionado ácido cítrico devido à acidez dos frutos. Foram realizados testes preliminares para a padronização da formulação da geleia, que foi única para os três estádios de colheita dos frutos.

A cocção foi feita em tacho de cobre aberto com capacidade máxima de $8 \mathrm{~L}$, com agitação manual contínua. A determinação do ponto final da geleia foi realizada com auxílio de refratômetro, fixando o valor de $65^{\circ}$ Brix como padrão.

As análises da geleia foram realizadas após 24 horas da fabricação para que houvesse estabilização da textura e da coloração. As avaliações realizadas foram: Umidade (Teor de Água): determinada pelo método de secagem em estufa através da perda de peso da amostra aquecida a $105^{\circ} \mathrm{C} \pm 1{ }^{\circ} \mathrm{C}$, até peso constante (Instituto Adolfo Lutz, 2008); Proteína bruta: calculada a partir dos teores de nitrogênio total, utilizando fator de conversão de 6,25. O nitrogênio total foi determinado pelo método Kjeldahl (HORWITZ, 2005), utilizando solução digestora com ácido sulfúrico em bloco de aquecimento; Açúcares (redutores, totais e sacarose): utilizou-se a metodologia descrita por Somogy (1945) e Nelson (1944). O aparelho utilizado foi o espectrofotômetro Micronal B 382, sendo a leitura realizada a $535 \eta \mathrm{m}$. Para o cálculo de sacarose, os teores de açúcares redutores são diminuídos dos teores de açúcares totais e multiplicados por um fator $(0,9)$. Lipídeos: determinados pelo método de extração Soxhlet, utilizando como extrator o éter de petróleo (HORWITZ, 2005); Fibra alimentar: a determinação foi realizada por diferença de peso dos papéis de filtro antes e após as digestões em metodologia descrita por Horwitz (2005); Pectina total, solúvel e porcentagem de solubilização: a extração das substâncias pécticas foi realizada segundo a técnica descrita por McCready e McComb (1952), e Minerais (N, P, K, Ca, Mg, S, B, Cu, Fe, Mn e $\mathrm{Zn}$ ): o material foi moído em moinho tipo "Willey" e encaminhado para o Laboratório de Nutrição Mineral de Plantas do Departamento de Recursos Naturais/Ciência do Solo, da FCA/UNESP, seguindo metodologia citada por Malavolta et al. (1997).

O experimento foi avaliado somente na colheita. Cada tratamento foi composto de cinco repetições formadas por bandejas com cerca de 200 $\mathrm{g}$ de frutas e potes de vidro com cerca de $200 \mathrm{~g}$ de geleia. Os resultados foram submetidos à análise de variância, e as médias, comparadas pelo teste de Tukey, ao nível de 5\% de probabilidade.

\section{RESULTADOS E DISCUSSÃO}

Os teores de umidade, proteína, açúcares redutores, lipídeos e fibra alimentar dos frutos estão dispostos na Tabela 1.

A amora-preta 'Tupy' apresentou alto conteúdo de água, conforme os resultados encontrados para os diferentes pontos de colheita. Nota-se que, quanto mais imaturo, maior a quantidade de água. Os valores encontrados para esta característica são semelhantes aos encontrados por Hirsh (2011), estudando diferentes cultivares de amora-preta, e o autor encontrou resultados que variaram de 84,8 a 90,3 $\mathrm{g}$ $100 \mathrm{~g}^{-1}$ nas cultivares 'Seleção 02/96' e 'Cherokee', respectivamente.

Os valores de proteína não apresentaram diferenças estatísticas entre si. Os experimentos de Hirsh (2011) mostram que, para as cultivares 'Tupy' e 'Seleção 03/01', os valores variaram de 0,09 a 0,14 g $100 \mathrm{~g}^{-1}$. Segundo Antunes (2002), o morango, o 
mirtilo e a framboesa apresentam baixos teores de proteínas e de gordura (em torno de 0,80 e 0,15 g 100 $\mathrm{g}^{-1}$, respectivamente, em base úmida), semelhantes aos encontrados no presente estudo.

O teor de açúcares redutores apresentou menor valor nos frutos colhidos precocemente. A polpa de amora-preta 'Tupy', estudada por Araújo (2009), apresentou menor teor de açúcares totais e redutores quando comparada à polpa deste estudo.

Os menores valores de lipídeo foram encontrados nos frutos colhidos precocemente, porém os tratamentos não se diferenciaram estatisticamente. Estes dados são semelhantes aos encontrados por Hirsh (2011). O autor encontrou resultados que va-

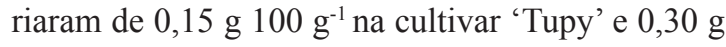
$100 \mathrm{~g}^{-1}$ na 'Seleção 02/96'.

Os frutos da amoreira-preta, assim como as demais frutas cuja película é consumida conjuntamente, apresentam teores de fibra bruta em torno 5\% (ARAÚJO, 2009). A fibra alimentar apresentou maiores resultados nos frutos precoces. Os teores de fibras brutas de amora assemelharam-se aos valores descritos por Araújo (2009), para polpa de amora-preta cv. 'Tupy', safra de 2007/2008.

Em relação à pectina total e solúvel, os resultados mostram que, quanto mais tardio o estádio de colheita, maior o teor de pectina total, enquanto a pectina solúvel apresenta aumento ao longo do tempo de armazenamento (Tabela 2). Nesta mesma tabela, são apresentados os valores de Porcentagem de Solubilização da Pectina presente nos frutos, sendo este parâmetro importante para o estudo do tempo de conservação dos frutos, já que quanto maior este valor, mais frágil a consistência dos mesmos. Estudos realizados por Antunes et al. (2006) mostram que, após a colheita, os frutos da cv. 'Brazos' apresentaram teores de pectina total de cerca de 600 $\mathrm{mg}$ de ác. galacturônico $100 \mathrm{~g}^{-1}$. Frutos com elevada porcentagem de pectina solúvel são geralmente de textura fraca e pouco resistentes ao transporte e armazenamento (CARVALHO, 1994), pois seu aumento reflete na degradação da parede celular e no amolecimento dos frutos. Além do armazenamento, a quantificação dos teores de pectina é importante, pois interfere na elaboração de geleia.

Quanto à umidade das geleias de amora-preta, todas as amostras dos diferentes tratamentos foram semelhantes aos recomendados pela norma vigente (BRASIL, 1978), que estabelece valores nutricionais dos alimentos (38\%). Mota (2006) encontrou valores de umidade que variaram de 43,22 a 49,82 g $100 \mathrm{~g}^{-1}$ para as cultivares 'Brazos' e 'Seleção 97', respectivamente.

As geleias apresentaram maiores teores de proteínas nos frutos tardios, apesar de não apresentarem diferença significativa em relação aos frutos colhidos precocemente.

Observou-se que todas as amostras apresentaram valores semelhantes para os teores de açúcares redutores. O mesmo ocorreu com relação aos teores de açúcar total.

Os teores de lipídeo das amostras avaliadas não apresentaram diferenças estatísticas, assim como os teores de fibras alimentares.

Os teores de umidade, proteína, açúcares redutores, lipídeos e fibra alimentar das geleias estão dispostos na Tabela 3 .

As frutas são exemplos de importantes fontes de elementos essenciais. Os minerais desempenham uma função vital no peculiar desenvolvimento e boa saúde do corpo humano e as frutas são consideradas as principais fontes de minerais necessários na dieta humana (HARDISSON, 2001).

Pode-se notar que a maioria dos minerais apresentou diminuição em seus teores conforme o estádio de maturação mais avançado dos frutos.

Quanto ao teor de cálcio $(\mathrm{Ca})$, os tratamentos não diferenciaram entre si, porém pôde-se notar leve tendência à queda. De acordo com Guedes et al. (2013), o maior teor de cálcio foi encontrado nas cultivares 'Cherokee' e 'Xavante', com 2,33 e 2,21 $\mathrm{g} \mathrm{kg}^{-1}$, respectivamente.

O nitrogênio $(\mathrm{N})$, o fósforo $(\mathrm{P})$, o potássio $(\mathrm{K})$ e o enxofre $(\mathrm{S})$ apresentaram valores semelhantes estatisticamente. Guedes et al. (2013), estudando diferentes cultivares de amora-preta, verificaram que as cultivares 'Choctaw', 'Tupy' e 'Xavante' apresentaram os maiores teores de fósforo $(1,99 ; 1,99$ e 1,96 $\mathrm{g} \mathrm{kg}^{-1}$ ), e o teor mais baixo foi observado na cultivar 'Ébano' (1,09 $\left.\mathrm{g} \mathrm{kg}^{-1}\right)$.

Quanto ao magnésio ( $\mathrm{Mg})$, Guedes et al. (2013), relatam a variação de $1,00 \mathrm{~g} \mathrm{~kg}^{-1}$ para a cultivar 'Ébano' a 2,14 $\mathrm{g} \mathrm{kg}^{-1}$ para a cultivar 'Choctaw', sendo o teor médio de $1,59 \mathrm{~g} \mathrm{~kg}^{-1}$, semelhantes aos encontrados neste experimento. O magnésio desempenha importante função na composição da molécula de clorofila; portanto, quanto maior a maturidade dos frutos, menores os níveis deste mineral (MALAVOLTA et al., 2002).

O cobre $(\mathrm{Cu})$ apresentou diferenças estatísticas entre os estádios de colheita dos frutos, sendo os frutos $100 \%$ vermelhos com os maiores teores.

Os teores de ferro (Fe) também apresentaram diferenças estatísticas entre os tratamentos, sendo que os frutos colhidos precocemente apresentaram maiores teores.

O manganês $(\mathrm{Mn})$ apresentou teores bem menores nos frutos $100 \%$ pretos, comparados aos 
demais tratamentos.

O Zinco (Zn) não apresentou diferenças estatísticas entre os estádios de colheita, porém observa-se tendência à diminuição com o aumento da maturação. Malavolta et al. (2002) descrevem o zinco como elemento envolvido na síntese e na degradação de açúcares.

Os teores de minerais em frutos são muito dependentes do solo, da fertilidade, das condições climáticas e cultivares (NOUR et al., 2011). Os resultados encontrados de macro e microminerais dos frutos de amora-preta, nos 3 estádios de colheita, são apresentados na Tabela 4 .

Comparando as Tabelas 4 (amoras-pretas) e 5 (geleias), pode-se notar que grande parte dos minerais analisados apresentaram diminuições. Isto pode ser explicado pelo fato de que, no processamento, quando as frutas passam pela despolpadeira, grande parte dos minerais ficam retidos nas sementes e na película. Outro fator importante é devido à quantidade de polpa de fruta utilizada na formulação da geleia ser de $50 \%$ do total dos ingredientes. Os valores de minerais das geleias de amora-preta estão dispostos na Tabela 5.

TABELA 1- Umidade (g $\left.100 \mathrm{~g}^{-1}\right)$, Proteínas (g 100g-1), Açúcar Redutor (g 100g-1), Lipídeos (g $\left.100 \mathrm{~g}^{-1}\right)$ e Fibra

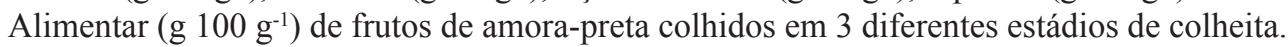

\begin{tabular}{|c|c|c|c|c|}
\hline & \multicolumn{3}{|c|}{ Frutos de amora-preta } & \multirow[b]{2}{*}{$\mathrm{CV}(\%)$} \\
\hline & $100 \%$ Vermelho & $50 \%$ vermelho, $50 \%$ preto & $100 \%$ preto & \\
\hline Umidade (g $\left.100 \mathrm{~g}^{-1}\right)$ & $90,22 \mathrm{a}$ & $87,62 \mathrm{ab}$ & $87,07 \mathrm{~b}$ & 1,9 \\
\hline Proteína (g $\left.100 \mathrm{~g}^{-1}\right)$ & $0,0976 \mathrm{a}$ & $0,1107 \mathrm{a}$ & $0,1191 \mathrm{a}$ & 12,97 \\
\hline Açúcar Redutor (g $\left.100 \mathrm{~g}^{-1}\right)$ & $6,9735 \mathrm{~b}$ & $8,5694 a$ & 8,6416 a & 23,13 \\
\hline Lipídeos (g $\left.100 \mathrm{~g}^{-1}\right)$ & $0,1320 \mathrm{a}$ & $0,1684 \mathrm{a}$ & $0,1575 \mathrm{a}$ & 85,47 \\
\hline 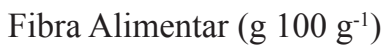 & $5,2944 \mathrm{a}$ & $4,8347 \mathrm{ab}$ & $4,1190 \mathrm{~b}$ & 9,13 \\
\hline
\end{tabular}

* Letras minúsculas diferentes na mesma linha indicam diferenças estatísticas entre cada amostra analisada $(\mathrm{p}<0,05)$ (teste de Tukey).

TABELA 2 - Pectina Solúvel, Total (mg de ác. galacturônico $100 \mathrm{~g}^{-1}$ ) e Porcentagem de Solubilização em frutos de amora-preta colhidos em 3 diferentes estádios de colheita.

\begin{tabular}{lcccc}
\hline \multicolumn{4}{c}{ Frutos de amora-preta } \\
\hline & $100 \%$ Vermelho & $50 \%$ vermelho, 50\% preto $100 \%$ preto & $\mathrm{CV}(\%)$ \\
\hline Pectina Solúvel (mg de ác. gal. $100 \mathrm{~g}^{-1}$ ) & $169,00 \mathrm{~b}$ & $347,33 \mathrm{a}$ & $494,67 \mathrm{a}$ & 16,82 \\
Pectina Total mg de ác. gal. $\left.100 \mathrm{~g}^{-1}\right)$ & $560,33 \mathrm{a}$ & $215,67 \mathrm{~b}$ & $182,00 \mathrm{~b}$ & 11,66 \\
$\%$ de Solubilização & $0,31 \mathrm{c}$ & $1,64 \mathrm{~b}$ & $2,73 \mathrm{a}$ & 23,00 \\
\hline
\end{tabular}

* Letras minúsculas diferentes na mesma linha indicam diferenças estatísticas entre cada amostra analisada ( $<<0,05)$ (teste de Tukey).

TABELA 3- Umidade (g $\left.100 \mathrm{~g}^{-1}\right)$, Proteínas (g $\left.100 \mathrm{~g}^{-1}\right)$, Açúcar Redutor (g $\left.100 \mathrm{~g}^{-1}\right)$, Açúcar Total (g 100 g Sacarose $\left(\mathrm{g} 100 \mathrm{~g}^{-1}\right)$, Lipídeos $\left(\mathrm{g} 100 \mathrm{~g}^{-1}\right)$ e Fibra Alimentar $\left(\mathrm{g} 100 \mathrm{~g}^{-1}\right)$ de geleias obtidas a partir de frutos de amora-preta colhidos em 3 diferentes estádios de colheita.

\begin{tabular}{|c|c|c|c|c|}
\hline & \multicolumn{3}{|c|}{ Geleia de amora-preta } & \multirow[b]{2}{*}{$\mathrm{CV}(\%)$} \\
\hline & $100 \%$ Vermelho & $50 \%$ vermelho, $50 \%$ preto & $100 \%$ preto & \\
\hline Umidade (g $\left.100 \mathrm{~g}^{-1}\right)$ & $36,4433 \mathrm{a}$ & $38,8338 \mathrm{a}$ & $37,6456 \mathrm{a}$ & 3,82 \\
\hline Proteína (g $\left.100 \mathrm{~g}^{-1}\right)$ & $0,0184 a b$ & $0,0130 \mathrm{~b}$ & 0,0193 a & 21,46 \\
\hline Açúcar Redutor (g $100 \mathrm{~g}^{-1}$ ) & $36,0930 \mathrm{a}$ & 37,7448 a & $34,9476 \mathrm{a}$ & 11,89 \\
\hline Açúcar Total (g $\left.100 \mathrm{~g}^{-1}\right)$ & $60,0899 \mathrm{a}$ & $61,8471 \mathrm{a}$ & $59,5441 \mathrm{a}$ & 12,84 \\
\hline Sacarose $\left(\mathrm{g} 100 \mathrm{~g}^{-1}\right)$ & $21,0706 \mathrm{a}$ & $21,6399 \mathrm{a}$ & $22,0972 \mathrm{a}$ & 6,18 \\
\hline Lipídeos (g $\left.100 \mathrm{~g}^{-1}\right)$ & $0,0980 \mathrm{a}$ & 0,0966 a & $0,1542 \mathrm{a}$ & 61,39 \\
\hline Fibra Alimentar (g $\left.100 \mathrm{~g}^{-1}\right)$ & $0,6558 \mathrm{a}$ & $0,5626 \mathrm{a}$ & $0,5468 \mathrm{a}$ & 26,51 \\
\hline
\end{tabular}

* Letras minúsculas diferentes na mesma linha indicam diferenças estatísticas entre cada amostra analisada $(\mathrm{p}<0,05)$ (teste de Tukey). 
TABELA 4- Cálcio $\left(\mathrm{g} \mathrm{kg}^{-1}\right)$, Fósforo $\left(\mathrm{g} \mathrm{kg}^{-1}\right)$, Potássio $\left(\mathrm{g} \mathrm{kg}^{-1}\right)$, Enxofre $\left(\mathrm{g} \mathrm{kg}^{-1}\right)$, Magnésio $\left(\mathrm{g} \mathrm{kg}^{-1}\right)$, Nitrogênio $\left(\mathrm{g} \mathrm{kg}^{-1}\right)$, Boro $\left(\mathrm{mg} \mathrm{kg}^{-1}\right)$, Cobre $\left(\mathrm{mg} \mathrm{kg}^{-1}\right)$, Ferro $\left(\mathrm{mg} \mathrm{kg}^{-1}\right)$, Manganês $\left(\mathrm{mg} \mathrm{kg}^{-1}\right)$ e Zinco (mg $\left.\mathrm{kg}^{-1}\right)$ de frutos de amora-preta colhidos em 3 diferentes estádios de colheita.

\begin{tabular}{lcccc}
\hline \multicolumn{4}{c}{ Frutos de amora-preta } \\
\hline Cálcio $\left(\mathrm{g} \mathrm{kg}^{-1}\right)$ & $100 \%$ Vermelho & $50 \%$ vermelho, $50 \%$ preto & $100 \%$ preto & $\mathrm{CV}(\%)$ \\
Fósforo $\left(\mathrm{g} \mathrm{kg}^{-1}\right)$ & $1,95 \mathrm{a}$ & $1,86 \mathrm{a}$ & $1,44 \mathrm{a}$ & 23,62 \\
Potássio $\left(\mathrm{g} \mathrm{kg}^{-1}\right)$ & $1,47 \mathrm{a}$ & $1,31 \mathrm{~b}$ & $1,31 \mathrm{~b}$ & 6,25 \\
Enxofre $\left(\mathrm{g} \mathrm{kg}^{-1}\right)$ & $8,34 \mathrm{a}$ & $7,46 \mathrm{a}$ & $8,08 \mathrm{a}$ & 7,06 \\
Magnésio $\left(\mathrm{g} \mathrm{kg}^{-1}\right)$ & $1,54 \mathrm{a}$ & $1,45 \mathrm{a}$ & $1,40 \mathrm{a}$ & 6,05 \\
Nitrogênio $\left(\mathrm{g} \mathrm{kg}^{-1}\right)$ & $1,88 \mathrm{a}$ & $1,86 \mathrm{a}$ & $1,72 \mathrm{a}$ & 7,23 \\
Boro $\left(\mathrm{mg} \mathrm{kg}^{-1}\right)$ & $14,40 \mathrm{a}$ & $14,88 \mathrm{a}$ & $14,65 \mathrm{a}$ & 10,86 \\
Cobre $\left(\mathrm{mg} \mathrm{kg}^{-1}\right)$ & $12,14 \mathrm{~b}$ & $22,75 \mathrm{a}$ & $22,30 \mathrm{a}$ & 13,29 \\
Ferro $\left(\mathrm{mg} \mathrm{kg}^{-1}\right)$ & $14,00 \mathrm{a}$ & $12,85 \mathrm{ab}$ & $11,40 \mathrm{~b}$ & 9,07 \\
Manganês $\left(\mathrm{mg} \mathrm{kg}^{-1}\right)$ & $96,20 \mathrm{a}$ & $48,40 \mathrm{ab}$ & $32,00 \mathrm{~b}$ & 9,67 \\
Zinco $\left(\mathrm{mg} \mathrm{kg}^{-1}\right)$ & $236,20 \mathrm{a}$ & $112,40 \mathrm{~b}$ & $69,40 \mathrm{~b}$ & 10,94 \\
\hline Letras minúsculas diferentes na mesma linha indicam diferenças estatísticas entre cada amostra analisada (p<0,05) (teste de Tukey).
\end{tabular}

TABELA 5 - Cálcio $\left(\mathrm{g} \mathrm{kg}^{-1}\right)$, Fósforo $\left(\mathrm{g} \mathrm{kg}^{-1}\right)$, Potássio $\left(\mathrm{g} \mathrm{kg}^{-1}\right)$, Enxofre $\left(\mathrm{g} \mathrm{kg}^{-1}\right)$, Magnésio $\left(\mathrm{g} \mathrm{kg}^{-1}\right)$, Nitrogênio $\left(\mathrm{g} \mathrm{kg}^{-1}\right)$, Boro $\left(\mathrm{mg} \mathrm{kg}^{-1}\right)$, Cobre $\left(\mathrm{mg} \mathrm{kg}^{-1}\right)$, Ferro $\left(\mathrm{mg} \mathrm{kg}^{-1}\right)$, Manganês $\left(\mathrm{mg} \mathrm{kg}^{-1}\right)$ e Zinco $\left(\mathrm{mg} \mathrm{kg}^{-1}\right)$ em geleias obtidas de frutos de amora-preta colhidos em 3 diferentes estádios de colheita.

\begin{tabular}{lcccc}
\hline \multicolumn{5}{c}{ Geleia de amora-preta } \\
\hline Cálcio $\left(\mathrm{g} \mathrm{kg}^{-1}\right)$ & $100 \%$ Vermelho & $50 \%$ vermelho, 50\% preto & $100 \%$ preto & $\mathrm{CV}(\%)$ \\
Fósforo $\left(\mathrm{g} \mathrm{kg}^{-1}\right)$ & $0,03 \mathrm{a}$ & $1,95 \mathrm{a}$ & $1,98 \mathrm{a}$ & 2,26 \\
Potássio $\left(\mathrm{g} \mathrm{kg}^{-1}\right)$ & $0,60 \mathrm{a}$ & $0,03 \mathrm{a}$ & $0,03 \mathrm{a}$ & 2,62 \\
Enxofre $\left(\mathrm{g} \mathrm{kg}^{-1}\right)$ & $1,03 \mathrm{a}$ & $0,51 \mathrm{ab}$ & $0,47 \mathrm{~b}$ & 12,7 \\
Magnésio $\left(\mathrm{g} \mathrm{kg}^{-1}\right)$ & $0,09 \mathrm{a}$ & $1,04 \mathrm{a}$ & $1,02 \mathrm{a}$ & 3,19 \\
Nitrogênio $\left(\mathrm{g} \mathrm{kg}^{-1}\right)$ & $0,45 \mathrm{a}$ & $0,09 \mathrm{a}$ & $0,09 \mathrm{a}$ & 3,31 \\
Boro $\left(\mathrm{mg} \mathrm{kg}^{-1}\right)$ & $1,58 \mathrm{a}$ & $0,27 \mathrm{a}$ & $0,14 \mathrm{a}$ & 35,39 \\
Cobre $\left(\mathrm{mg} \mathrm{kg}^{-1}\right)$ & $11,96 \mathrm{a}$ & $1,76 \mathrm{a}$ & $1,37 \mathrm{a}$ & 22,14 \\
Ferro $\left(\mathrm{mg} \mathrm{kg}^{-1}\right)$ & $22,07 \mathrm{a}$ & $6,53 \mathrm{~b}$ & $2,66 \mathrm{~b}$ & 56,1 \\
Manganês $\left(\mathrm{mg} \mathrm{kg}^{-1}\right)$ & $5,85 \mathrm{a}$ & $7,67 \mathrm{~b}$ & $7,02 \mathrm{~b}$ & 47,4 \\
Zinco $\left(\mathrm{mg} \mathrm{kg}^{-1}\right)$ & $2,27 \mathrm{a}$ & $0,98 \mathrm{~b}$ & $0,96 \mathrm{~b}$ & 61,59 \\
\hline
\end{tabular}

* Letras minúsculas diferentes na mesma linha indicam diferenças estatísticas entre cada amostra analisada $(\mathrm{p}<0,05)$ (teste de Tukey). 


\section{CONCLUSÃO}

Os resultados do experimento mostram que o ponto de colheita das frutas $100 \%$ vermelhas apresenta valores superiores na maioria dos parâmetros avaliados, tanto para as frutas quanto para as geleias, demonstrando maior perda destes compostos quanto mais tardia a colheita. A amora-preta mostra ser um fruto bastante versátil, possuindo características interessantes, tanto para seu consumo in natura quanto para seu processamento.

\section{REFERÊNCIAS}

ANTUNES, L. E. C. Amora-preta: nova opção de cultivo no Brasil. Ciência Rural, Santa Maria, v. 32, p. 151-158, 2002.

ANTUNES, L. E. C.; GONÇALVES, E. D.; TREVISAN, R. Alterações da atividade da Poligalacturonase Pectinametilesterase em amorapreta (Rubus spp.) durante o armazenamento. Revista Brasileira de Agrociência, Pelotas, v. 12, n. 1, p. 63-66, 2006.

ARAÚJO, P. F. Atividade antioxidante de néctar de amora-preta (Rubus spp.) e sua influência sobre os lipídios séricos, glicose sanguínea e peroxidação lipídica em hamsters (Mesocricetus auratus) hipercolesterolêmicos. 2009. 123f. Dissertação (Mestrado em Ciência e Tecnologia Agroindustrial) - Universidade Federal de Pelotas, Pelotas, 2009.

BRASIL. Ministério da Saúde. Secretaria de Vigilância Sanitária. Normas Técnicas Especiais do Estado de São Paulo, relativas a alimentos e bebidas. Resolução da Comissão Nacional de Normas e Padrões para Alimentos - CNNPA n. 12, de 24 de julho de 1978. Seção I, pt I. Disponível em:

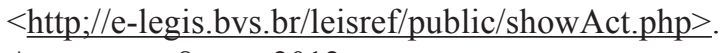
Acesso em: 8 mar. 2012.

CARVALHO, V. D. Qualidade e conservação póscolheita de goiabas. Informe Agropecuário, Belo Horizonte, v.17, n. 179, p. 48-54, 1994.

DEIGHTON, N.; BRENNAN, R.; FINN, C.; DAVIES, H. V. Antioxidant properties of domesticated and wild Rubus species. Journal of the Science of Food and Agriculture, Dundee, v.80, p. 1307-1313, 2000.
GUEDES, M. N. S.; ABREU, C. M. P.; MARO, L. A. C.; PIO, R.; ABREU, J. R.; OLIVEIRA, J. O. Chemical characterization and mineral levels in the fruits of blackberry cultivars grown in a tropical climate at an elevation. Acta Scientiarum. Agronomy, Maringá, v. 35, n. 2, p. 191-196, 2013.

HARBORNE, J. B.; WILliANS, C. A. Advances in flavonoid research since 1992. Phytochemistry, Maryland, v. 55, p. 481-504, 2000.

HARDISSON, A.; RUBIO, A; BAEZ, A.; MARTIN, M.; ALVAREZ, R.; DIAZ, E. Mineral composition of the banana (Musa acuminata) from the island of Tenerife. Food Chemistry, London, v. 73, n.1, p. 153-161, 2001.

HIRSH, G. E. Valor nutricional e capacidade antioxidante de diferentes genótipos de amorapreta (Rubu ssp). 2011. 100 f. Dissertação (Mestrado Ciência e Tecnologia de Alimentos) - Universidade Federal de Santa Maria, Santa Maria, 2011.

HORWITZ, W. Official methods of analysis. Gaithersburg: AOAC International, 2005. v.18.

INSTITUTO ADOLFO LUTZ. Normas analíticas do Instituto Adolfo Lutz: métodos químicos e físicos para análise de alimentos. 4.ed. São Paulo: IMESP, 2008.

KOCA, I.; KARADENIZ, B. Antioxidant properties of blackberry and blueberry fruits grown in the Black Sea Region of Turkey. Scientia Horticulturae, Amsterdam, v.121, n.4, p.447-450, 2009.

MALAVOLTA, E.; GOMES, F. P.; ALCARDE, J. C. Adubos e adubações. São Paulo: Nobel, 2002. 200 p.

MALAVOLTA, E.; VITTI, G. C.; OLIVEIRA, S. A. Avaliação do estado nutricional das plantas: princípios e aplicações. Piracicaba: Potafós, 1997. 319 p.

MCCREADY, P. M.; MCCOMB, E. A. Extraction and determination of total pectic material. Analytical Chemistry, Washington, v. 24, n. 12, p. 1586-1588, 1952. 
MOTA, R. V. Caracterização física e química de geleia de amora-preta. Ciência e Tecnologia de Alimentos, Campinas, v.26, n.3, p.539-543, 2006.

MOYER, R. A.; HUMMER, K. E.; FINN, C. C.; FREI, B.; WROLSTAD, R. E. Anthocyanins, phenolics, and Antioxidants capacity in diverses mallfruits: Vaccinium, Rubus, and Ribes. Journal of Agricultural and Food Chemistry, Tennessee, v. 50, p. 519-525, 2002.

NELSON, N. A. A photometric adaptation of Somogy method for the determination of glucose. Journal Biological Chemistry, Bethesda, v. 153, p. 375-380, 1944.

NOUR, V.; TRANDAFIR, I.; IONICA, M. E. Ascorbic acid, anthocyanins, organic acids and mineral content of some black and red currant cultivars. Fruits, Paris, v. 66, n.5, p. 353-362, 2011.
SOMOGY, M. Determination of blooded sugar. Journal Biologic Chemical, Baltimore, n.160, p. 69-73, 1945.

VIZZOTO, M. Amora-preta: uma fruta antioxidante. Pelotas: Embrapa Clima Temperado, 2008.

WANG, S.Y.; LIN, H.S. Antioxidant activity in fruits and leaves of blackberry, raspberry, and strawberry varies with cultivar and developmental stage. Journal of Agricultural and Food Chemistry, Maryland, v. 48, p. 140-146, 2000. 